\title{
Clinical implication of hepatic volumetry for living donor liver transplantation
}

\author{
Woo Kyoung Jeong \\ Department of Radiology and Center for Imaging Sciences, Samsung Medical Center, Sungkyunkwan University School of Medicine, \\ Seoul, Korea
}

Keywords: Computed Tomography; Volumetry; Liver Transplantation; Small-for-size-graft

\section{See Article on Page 54}

Liver transplantation is indicated for primary and salvage treatment of hepatic malignancies including hepatocellular carcinoma ( $\mathrm{HCC}$ ) and hepatic epithelioid hemangioendothelioma, end-stage liver diseases, acute liver failure, and congenital anatomical and metabolic liver diseases such as biliary atresia, Wilson's disease and hemochromatosis.' Living donor liver transplantation (LDLT) is more widely practiced in many Asian countries, rather than in Europe and the US, due to shortage of donors who have suffered brain death. In Korea, $75.4 \%$ of LT by 2016 was LDLT. ${ }^{2}$ Pre-operative hepatic volumetry by using multi-detector row computed tomography (CT) or magnetic resonance imaging (MRI) is one of the most important step during the planning of $\mathrm{LDLT}^{3}$ because hepatic reservoir after surgery could be estimated by the hepatic volumetry, which is an important factor for donor's and recipient's recovery after surgery. ${ }^{4,5}$

If the graft size is too small, and mismatched compared with the recipient's size, it is called as "small-for-size-graft (SFSG)". In that situation, even the small graft could be well adapted, but some recipient could have symptoms related to the graft size. ${ }^{6}$ SFSG dysfunction was defined as 'dysfunction of a small partial liver graft during the first postoperative week after the exclusion of other causes.'. It means that the transplanted partial liver cannot satisfy metabolic demand of the recipient or can be prone to congest by excessive portal inflow before regeneration of the graft, and results in irreversible graft failure and death of the patient if there is no available organ for re-transplantation. In the general setting of LDLT, the graft volume of less than $40-50 \%$ of standardized liver volume (SLV), corresponding to a graft to recipient weight ratio (GRWR) of $0.8-1.0 \%$ could be associated with worse outcome. ${ }^{8}$ The mechanism of SFSG syndrome is not clear. It could be related to the shortage of parenchymal cell mass required to metabolize, and persistent elevation of portal venous pressure which causes hyperperfusion graft injury. ${ }^{6}$ SFSG get the entire volume of portal flow, and lead to higher portal pressure due to increased portal resistance. ${ }^{9}$ Moreover, as the cirrhotic recipients demonstrate higher portal flow than normal subjects, this recipients' hemodynamics

\section{Abbreviations:}

$C T$, computed tomography; GRWR, graft to recipient weight ratio; HCC, hepatocellular carcinoma; LDLT, Living donor liver transplantation; MRI, magnetic resonance imaging; SFSG, small-for-size-graft, SLV, standardized liver volume

\section{Corresponding author: Woo Kyoung Jeong}

Department of Radiology and Center for Imaging Science, Samsung Medical Center, Sungkyunkwan University School of Medicine, 81 Irwon-ro, Gangnam-gu, Seoul 06351, Korea

Tel: +82-2-3410-1923, Fax: +82-2-3410-0049

E-mail:jeongwk@gmail.com

https://orcid.org/0000-0002-0676-2116

Received : Feb. 14, 2018 / Accepted : Feb. 22, 2018 
with excessive portal flow is also important in the setting of partial transplantation. This situation can result in endothelial injury of hepatic sinusoids and damage of hepatocytes. According to the human study for SFSG syndrome, $80 \%$ of patients who had a graft volume of less than $40 \%$ of SLV showed the morphological hallmarks, accompanied by gaps in the sinusoidal endothelial lining and mitochondrial swelling as well as vacuolar changes in hepatocytes on electron microscopy. ${ }^{10}$

Especially, hepatic steatosis is spreading gradually and it is an important risk factor for post-operative complications after major hepatectomy and LDLT. ${ }^{7,11}$ Though the precise effect of hepatic steatosis on graft function has not been understood yet, it is prudent to aim for a larger hepatic volume when considering using the partial liver with steatosis because the immediate functional capacity of the graft is important to tolerate the recipient.

As mentioned previously, hepatic reservoir is important to recover from post-operative state, and almost all transplantation centers measure the hepatic volume using pre-operative imaging studies. CT volumetry was first performed with cadaveric experiment in 1979, and the accuracy was higher than $95 \% .^{12}$ The conventional method of segmentation for hepatic volumetry uses the anatomic landmarks according to Couinaud classification ${ }^{13}$ : the imaginary plane between middle hepatic vein and the fossa for gallbladder divides into both hemilivers, and middle hepatic vein and its tributaries to segment IV are not included in the graft because they should be remained at operation for donor safety.

Kwon, et al. performed the hepatic volumetry using pre- and post-operative CT imaging data, and addressed that there was about $5 \%$ of discrepancy between the estimated volume measured by conventional method which divides as the anatomic landmark (prospective volumetry on pre-operative CT) and the volume of the graft measured after transplantation (retrospective volumetry on post-operative (T), and about 10\% error between prospective volume and the weight of graft measured at operation. ${ }^{14}$ It suggests the pre-operative volumetry could be overestimated compared with the weight of procured graft, and it is caused by the blood volume circulating in the large hepatic vessels.

These errors should be handled with caution, especially in the recipient with SFSG. The authors raised the issue which should be considered at pre-operative stage. As solutions to compensate, they mentioned applying an accurate conversion factor and computer-aided liver volumetry that can assess the bloodless liver volume. ${ }^{15}$ In addition, the author highlighted that experienced operator should perform the volumetry to reduce the amount of error. The knowledge about hepatic anatomy and the communica- tion to the surgeon are still necessary for accurate measurement.

Recent volumetric technique has been improved by various segmentation techniques with 3D-image data, such as manual segmentation with assisted contouring and assisted in-painting technique, intensity-based semi-automatic segmentation, graphcut technique, and fully automated segmentation using statistical shape models and 3-D deformable models. ${ }^{3}$ These novel techniques will provide the accurate and fast result of volumetry to the surgeons in the near future.

\section{Conflicts of Interest}

The authors have no conflicts to disclose.

\section{REFERENCES}

1. European Association for the Study of the Liver. EASL Clinical Practice Guidelines: Liver transplantation. J Hepatol 2016;64:433-485.

2. Korean Network for Organ Sharing (KONOS). Yearly statistical information of liver transplantation in Korea 2000-2016. KONOS Web site <https://www.konos.go.kr/konosis/common/bizlogic.jsp\#>. Accessed 2016.11.02.

3. Gotra A, Sivakumaran L, Chartrand G, Vu KN, Vandenbroucke-Menu F, Kauffmann C, et al. Liver segmentation: indications, techniques and future directions. Insights Imaging 2017;8:377-392.

4. Fan ST, Lo CM, Liu CL, Yong BH, Chan JK, Ng IO. Safety of donors in live donor liver transplantation using right lobe grafts. Arch Surg 2000;135:336-340.

5. Ben-Haim M, Emre S, Fishbein TM, Sheiner PA, Bodian CA, KimSchluger $L$, et al. Critical graft size in adult-to-adult living donor liver transplantation: impact of the recipient's disease. Liver Transpl 2001;7:948-953.

6. Kiuchi T, Tanaka K, Ito T, Oike F, Ogura Y, Fujimoto Y, et al. Small-forsize graft in living donor liver transplantation: how far should we go? Liver Transpl 2003;9:S29-S35.

7. Dahm F, Georgiev P, Clavien PA. Small-for-size syndrome after partial liver transplantation: definition, mechanisms of disease and clinical implications. Am J Transplant 2005;5:2605-2610.

8. Kiuchi T, Kasahara M, Uryuhara K, Inomata Y, Uemoto S, Asonuma $K$, et al. Impact of graft size mismatching on graft prognosis in liver transplantation from living donors. Transplantation 1999;67:321327.

9. Ito T, Kiuchi T, Yamamoto H, Oike F, Ogura Y, Fujimoto Y, et al. Changes in portal venous pressure in the early phase after living donor liver transplantation: pathogenesis and clinical implications. Transplantation 2003;75:1313-1317.

10. Man K, Fan ST, Lo CM, Liu CL, Fung PC, Liang TB, et al. Graft injury in relation to graft size in right lobe live donor liver transplantation: a 
study of hepatic sinusoidal injury in correlation with portal hemodynamics and intragraft gene expression. Ann Surg 2003;237:256264.

11. Cho JY, Suh KS, Kwon CH, Yi NJ, Cho SY, Jang JJ, et al. The hepatic regeneration power of mild steatotic grafts is not impaired in livingdonor liver transplantation. Liver Transpl 2005;11:210-217.

12. Heymsfield SB, Fulenwider T, Nordlinger B, Barlow R, Sones P, Kutner $M$. Accurate measurement of liver, kidney, and spleen volume and mass by computerized axial tomography. Ann Intern Med 1979;90:185-187.

13. Vauthey JN, Zimmitti G, Shindoh J. From Couinaud to molecular biology: the seven virtues of hepato-pancreato-biliary surgery. HPB (Oxford) 2012;14:493-499.

14. Kwon HJ, Kim KW, Kim B, Kim SY, Lee CS, Lee J, et al. Resection plane-dependent error in computed tomography volumetry of the right hepatic lobe in living liver donors. Clin Mol Hepatol 2017;24:5460.

15. Kim KW, Lee J, Lee H, Jeong WK, Won HJ, Shin YM, et al. Right lobe estimated blood-free weight for living donor liver transplantation: accuracy of automated blood-free CT volumetry--preliminary results. Radiology 2010;256:433-440. 